Supporting Information for:

\title{
Orienting Block Copolymer Thin Films via Entropy
}

Ting-Ya Lo ${ }^{1}$, Ashkan Dehghan ${ }^{2}$, Prokopios Georgopanos ${ }^{3}+$, Apostolos Avgeropoulos ${ }^{3}$, An-Chang Shi ${ }^{2}$ * and Rong-Ming $\mathrm{Ho}^{l *}$

${ }^{1}$ Department of Chemical Engineering, National Tsing Hua University, Hsinchu 30013, Taiwan, R.O.C.

${ }^{2}$ Department of Physics and Astronomy, McMaster University, Hamilton, Ontario, Canada

${ }^{3}$ Department of Materials Science Engineering, University of Ioannina, University Campus, Ioannina 45110, Greece

$\dagger$ Present address: Helmholtz-Zentrum Geesthacht, Institute of Polymer Research, Max-Planck-Straße 1, 21502 Geesthacht, Germany 
To investigate the wettability of the constituted block on different interfaces, the interfacial energy differences between PS and PDMS for the substrate $\left(\gamma\right.$ PDMS-sub $\left.{ }^{2800 \mathrm{C}}-\gamma_{\mathrm{PS}-\text { sub }}{ }^{2800 \mathrm{C}}\right)$ and air surface $\left(\gamma_{\mathrm{PS}-}\right.$ air $^{2800 \mathrm{C}}-\gamma_{\mathrm{PDMS}-\text { air }}{ }^{2800 \mathrm{C}}$ ) were estimated. It is well documented that the surface tension of polymers varies linearly with temperature with a slope given by $\mathrm{d} \gamma / \mathrm{dT} .{ }^{1,2}$ Consequently, the surface tensions of the PS and PDMS at $280^{\circ} \mathrm{C}$ were estimated using the $\mathrm{d} \gamma_{\mathrm{PS}} / \mathrm{dT} \sim-0.076\left(\mathrm{mN} / \mathrm{m}^{\circ} \mathrm{C}\right)^{3}$ and $\mathrm{d} \gamma_{\mathrm{PDMS}} / \mathrm{dT} \sim-0.048$ $\left(\mathrm{mN} / \mathrm{m}^{\circ} \mathrm{C}\right),{ }^{4}$ respectively. Table $\mathrm{S} 1$ summarize the polar and dispersion components of the surface energies for the PS and PDMS homopolymers at room temperature and at $280^{\circ} \mathrm{C}$

Table S1. $\gamma^{d}$ and $\gamma^{p}$ values for the PS and PDMS materials at room temperature and $280^{\circ} \mathrm{C}$.

\begin{tabular}{ccccc}
\hline & \multicolumn{2}{c}{ Room temperature } & \multicolumn{2}{c}{ At $280^{\circ} \mathrm{C}$} \\
\hline Materials & $\gamma^{d}(\boldsymbol{m} \boldsymbol{N} / \boldsymbol{m})$ & $\gamma^{p}(\boldsymbol{m} \boldsymbol{N} / \boldsymbol{m})$ & $\gamma^{d}(\boldsymbol{m} \boldsymbol{N} / \boldsymbol{m})$ & $\gamma^{p}(\boldsymbol{m} \boldsymbol{N} / \boldsymbol{m})$ \\
& & & & \\
\hline $\mathrm{SiO} 2$ & 21.9 & 50 & 21.9 & 50 \\
PDMS & 19 & 0.8 & 6.7 & 0 \\
PS & 33.9 & 6.8 & 14.4 & 0
\end{tabular}

Meanwhile, the interfacial energies of PS and PDMS for the substrate upon thermal annealing at $280^{\circ} \mathrm{C}$ were estimated as ${ }^{1,2}$

$$
\gamma_{12}=\gamma_{1}+\gamma_{2}-\mathrm{W}_{12}
$$

Where $\gamma_{1}$ is the surface tension of PS or PDMS, $\gamma_{2}$ is the surface tension of the $\mathrm{SiO}_{2}$ substrate, and $\mathrm{W}_{12}$ is the work of adhesion of PS or PDMS with the $\mathrm{SiO}_{2}$ substrate. The work of adhesion $\left(\mathrm{W}_{12}\right)$ of PS or PDMS with the $\mathrm{SiO}_{2}$ substrate was obtained using the geometric-mean equation as ${ }^{1,2}$

$$
W_{12}=2\left(\gamma_{1}^{d} \gamma_{2}^{d}\right)^{\frac{1}{2}}+2\left(\gamma_{1}^{p} \gamma_{2}^{p}\right)^{\frac{1}{2}}
$$


Where the terms with superscript $p$ and $d$ are referred to the polar and dispersion components of surface tensions, respectively. Consequently, the interfacial energy of PS or PDMS for the substrate can be calculated by

$$
\begin{array}{cc}
\gamma_{P D M S-S i O 2}{ }^{280^{\circ} \mathrm{C}}=\gamma_{P D M S}{ }^{280^{\circ} \mathrm{C}}+\gamma_{\mathrm{SiO} 2}{ }^{280^{\circ} \mathrm{C}}-W_{P D M S-S i O 2}{ }^{280^{\circ} \mathrm{C}} & \mathrm{Eq}[3] \\
\gamma_{P S-\mathrm{SiO} 2}{ }^{280^{\circ} \mathrm{C}}=\gamma_{P S}{ }^{280^{\circ} \mathrm{C}}+\gamma_{\mathrm{SiO} 2}{ }^{280^{\circ} \mathrm{C}}-W_{P S-\mathrm{SiO} 2}{ }^{280^{\circ} \mathrm{C}} & \mathrm{Eq}[4]
\end{array}
$$

Finally the $\Delta \gamma$ value for the substrate and for the air surface were calculated as $\Delta \gamma_{\text {sub }}=$ $\gamma_{P D M S-s u b^{280^{\circ} C}}-\gamma_{P S-s u b^{280^{\circ} C}}=3.6(\mathrm{mN} / \mathrm{m})$ and $\Delta \gamma_{\text {air }}=\gamma_{P S-a i r^{280^{\circ} C}}-\gamma_{P D M S-a i r^{280^{\circ} C}}=7.7(\mathrm{mN} / \mathrm{m})$, respectively.

\section{Theoretical Framework}

In this section, we present a brief description of the Self-Consistent Field Theory (SCFT). A more comprehensive description of the SCFT formalism can be found in the literature. ${ }^{5,6}$ In what follows, we use linear AB-diblock copolymers as a model system to describe the SCFT. Generalizing the theory to $(\mathrm{AB})_{\mathrm{n}}$ star-block copolymers is straightforward. We consider a system of $\mathrm{n}$ AB-diblock copolymers of length $\mathrm{N}$, in a thin film of height $\mathrm{h}$. The monomer-monomer interactions are modeled using the Flory-Huggins parameter $\chi$. We will also introduce a parameter $h_{\boldsymbol{a}}(\mathrm{r})$ to control the polymer/substrate and polymer/air interactions, where the subscript $\alpha=\{A, B\}$. The asymmetry in the air and substrate interactions is modeled by allowing $h_{\alpha}(r)$ to be different for each polymer species.

We define the densities of the polymers at position $r$ in the system as

$$
\begin{aligned}
& \phi_{A}(r)=\frac{1}{\rho} \sum_{i=1}^{n} \int_{0}^{f_{A} N} \delta\left(r-R_{i}^{A}(s)\right), \\
& \phi_{B}(r)=\frac{1}{\rho} \sum_{i=1}^{n} \int_{0}^{f_{B} N} \delta\left(r-R_{i}^{B}(s)\right),
\end{aligned}
$$


where $f_{A}$ and $f_{B}$ are the fractions of the $A$ and $B$ in AB-diblock copolymers. In the above equation, $R_{i}^{A}(s)$ and $R_{i}^{B}(s)$ are space curves determining the position of polymer segment $s$ in the system. In our model, we assume that all polymer species have the same monomer density $\rho$.

The thermodynamic properties of the system can be most conveniently described using a canonical ensemble at fixed temperature, volume and concentration. Using the above definitions, we can write the partition function as

$$
Z=\frac{1}{n !} \int \prod_{i=1}^{n} D\left[R_{i}^{A B}(s)\right] P\left[R_{i}^{A B}(s)\right] e^{-\beta E[\phi]} \prod_{r} \delta\left[\phi_{A}(r)+\phi_{B}(r)-1\right], \quad \mathrm{Eq}[3]
$$

where the delta function is introduced to ensure the incompressibility. Here we assume that polymers are flexible Gaussian chains, with Weiner measure given by

$$
P\left[R_{i}^{A B}(s)\right] \propto \exp \left[\frac{-3}{2 b^{2}} \int_{0}^{N} d s\left(\frac{d R_{i}^{A B}(s)}{d s}\right)^{2}\right]
$$

Here, $b$ is the Kuhn length of the polymers, which we assume to be the same for all polymer species.

The energy of the system is consist of two parts $E[\phi]=W[\phi]+H[\phi]$, where $W[\phi]$ is the energy due to the local interactions between the polymers and $H[\phi]$ captures the interactions between the polymers and the air/substrate surfaces. We can write $E[\phi]$ as

$$
E[\phi]=\int d r \chi_{A B} \phi_{A} \phi_{B}+\sum_{\alpha=A, B} \int d r h_{\alpha}(r) \phi_{\alpha}(r)
$$

where, $\mathrm{h}(\mathrm{r})$ controls the polymer/air and polymer/substrate interactions. We assume that surface interactions are short range and write, $h_{\alpha}(r)=\chi_{\alpha}^{\text {Air }}$ at the air surface, $h_{\alpha}(r)=\chi_{\alpha}^{\text {Sub }}$ at the substrate and $h_{\alpha}(r)=0$ otherwise. We apply the SCFT formalism [1-2], and rewrite the partition function as

$$
\begin{gathered}
Z=\int \prod_{\alpha=A, B} D\left[\phi_{\alpha}(r)\right] D\left[\omega_{\alpha}(r)\right] \prod_{r} \delta\left[\phi_{A}(r)+\phi_{B}(r)\right. \\
-1] \exp \left[\frac{-F[\phi, \omega]}{k T}\right],
\end{gathered}
$$

where, $\omega_{\alpha}(r)$ are the auxiliary fields. In the above equation, the free energy functional is defined as 


$$
\begin{aligned}
& \frac{F[\phi, \omega]}{k T \rho}=\int d r \chi_{A B} \phi_{A}(r) \phi_{B}(r) \\
& +\sum_{\alpha=A, B}\left[\int d r h_{\alpha}(r) \phi_{\alpha}(r)\right. \\
& \left.\quad-\int d r \omega_{\alpha}(r) \phi_{\alpha}(r)\right]-V \frac{1}{N} \ln \left(\frac{Q_{A B}}{\phi}\right),
\end{aligned}
$$

where $Q_{A B}$ is the single chain partition function of the AB-diblock copolymers. We can write $Q_{A B}$ as

$$
\begin{aligned}
Q_{A B}=\frac{1}{V} \int D\left[R_{i}^{A B}(s)\right] P\left[R_{i}^{A B}(s)\right] \delta\left[R_{i}^{A}\left(N_{A}\right)\right. \\
\left.\quad-R_{i}^{B}\left(N_{B}\right)\right] \exp \left[-\sum_{\alpha=A, B} \int_{0}^{N_{\alpha}} d s \omega_{\alpha}\left(R_{i}^{\alpha}(s)\right)\right] .
\end{aligned}
$$

Here, the delta function ensures the connectivity of the $\mathrm{A} / \mathrm{B}$ blocks. We will also rewrite the incompressibility condition given in equation [6] by using the identity

$$
\begin{aligned}
\prod_{r} \delta\left[\phi_{A}(r)+\right. & \left.\phi_{B}(r)-1\right] \\
& =\int D[\eta(r)] \exp \left[\int d r \eta(r)\left(\phi_{A}(r)+\phi_{B}(r)-1\right)\right], \quad \mathrm{Eq}[9]
\end{aligned}
$$

where $\eta(r)$ is a Lagrange multiplier.

We can determine the mean field solutions to the free energy functional by demanding that it is stationary with respect to variations in $\phi_{\alpha}(r), \omega_{\alpha}(r)$ and $\eta(r)$ fields. This results in the following set of self-consistent field equations 


$$
\begin{gathered}
\phi_{A}(r)=\frac{1}{Q_{A B} N} \int_{0}^{f_{A} N} d s q_{A}(r, s) q_{A}^{\dagger}\left(r, f_{A} N-s\right), \\
\phi_{B}(r)=\frac{1}{Q_{A B} N} \int_{0}^{f_{B} N} d s q_{B}(r, s) q_{B}^{\dagger}\left(r, f_{B} N-s\right), \\
\omega_{A}(r)=\chi_{A B} \phi_{B}(r)+h_{A}(r)+\eta(r), \\
\omega_{B}(r)=\chi_{A B} \phi_{A}(r)+h_{B}(r)+\eta(r), \\
\phi_{A}(r)+\phi_{B}(r)=1 .
\end{gathered}
$$

Here, $q_{\alpha}(r, s)$ and $q_{\alpha}^{\dagger}(r, s)$ are the forward and complementary end-integrated propagators. The propagators can be determine by solving the modified diffusion equations

$$
\begin{aligned}
& \frac{\partial q_{\alpha}(r, s)}{\partial s}=\frac{b_{\alpha}^{2} N}{6} \nabla^{2} q_{\alpha}(r, s)-\omega_{\alpha}(r), \\
& \frac{\partial q_{\alpha}^{\dagger}(r, s)}{\partial s}=\frac{b_{\alpha}^{2} N}{6} \nabla^{2} q_{\alpha}^{\dagger}(r, s)-\omega_{\alpha}(r) .
\end{aligned}
$$

We solve the modified diffusion equations using the real-space pseudo-spectral technique with the appropriate initial and boundary conditions.

\section{$\underline{\text { SCFT Calculations }}$}

The effects of block copolymer architecture on the orientation of lamellae- and cylinder-forming BCPs were investigated using Self-Consistent Field Theory (SCFT) calculations. Specifically, the phase behavior of a series of $n$-armed AB star-block copolymers with $n=1,2,3$ and 4 (each molecule is formed by joining $\mathrm{n}$ diblocks together with their A-ends) was studied using SCFT. The case of $\mathrm{n}=1$ corresponds to a linear AB-diblock copolymer. In our model, the A-species interact favorably with the air surface and tend to wet the polymer-air interface. The volume fraction of the A and B blocks and the A-B interaction were chosen such that the n-armed star-block copolymers form lamellar and cylinder phases in bulk. We examined the phase behavior of the n-armed $A B$ star-block copolymers confined to thin films of 
thickness $h \approx 2 d_{0}$ and $3 d_{o}$, where $d_{o}$ is the period of the bulk lamellar and cylinder phases for the $n$-armed star-block copolymers. The thickness of the film was chosen such that there would be no structural frustration due to film thickness. ${ }^{7}$ It should be pointed out that, due to the limitation of computational power, the film thickness used in the SCFT calculations is much smaller than the experimental values. Also, it is important to note that the polymer/air and polymer/substrate interaction parameters are chosen to approximate the experimentally measured surface interactions. As a result, the comparison of the theoretical results to experiments is qualitative. Instead, the theoretical study reveals the origin of the entropic effect is the topology of the star-copolymers. The relative polymer-substrate and polymer-air surface interactions were selected to resemble those of the experimental system. Specifically, $\Delta \chi=\chi_{\text {BAir }-}$ $\chi_{\text {AAir }}$ was chosen as a controlling parameter to tune the relative interaction between air and the A-B species, while the parameters $\chi_{\mathrm{ASub}}, \chi_{\mathrm{BSub}}, \chi_{\mathrm{AB}}, f_{\mathrm{B}}$ and the film thickness $\mathrm{h}$ were fixed. For both lamellaeand cylinder-forming systems, we consider the free energies of the parallel, perpendicular and mixed morphologies, where the density profiles for the morphologies studied are shown in Figure S5. In the parallel/perpendicular phases, for both lamellae- and cylinder-forming systems, microdomains are formed parallel/perpendicular to the air and substrate interfaces, respectively. In the mixed phase however, owing to the large polymer-air interaction, microdomains are formed parallel to the air surface at the air interface and perpendicular in the bulk region of the film initiated from the substrate.

Morphological transitions between parallel and perpendicularly oriented nanostructures were determined by comparing their free energies calculated from SCFT calculations. In Figure S6 and S7, we show the free energy of the parallel, perpendicular and mixed morphologies for the lamellae- and cylinder-forming 1-, 2-, 3- and 4-arm star-block copolymers as a function of $\Delta \chi$. For both lamellae- and cylinder-forming systems, the perpendicular phase has the lowest free energy at low $\Delta \chi$ values. In this region, the difference between the A- and B-air surface interactions is small, and thus we expect the perpendicular phase to be the stable structure. As $\Delta \chi$ is increased, a phase transition from the perpendicular to parallel phase in both lamella- and cylinder-forming systems can be observed. Note that for both lamella- and cylinder-forming systems, the mixed morphology is a metastable phase with 
free energy greater than the perpendicular and parallel phases. We will now examine the critical value of $\Delta \chi$ at which this transition occurs. As the difference in polymer-air interaction between the A and B species is increased $(\Delta \chi$ is increased), it becomes energetically more favorable to orient the microdomains in the parallel configuration. However, there is an entropic penalty for reorienting the microdomains parallel to the surfaces. There exists a critical $\Delta \chi$ beyond which the enthalpic contribution to the free energy outweighs the entropic effect and the parallel phase has the lower free energy. Owing to the entropic penalty for orienting the microdomains parallel to the surfaces for star-block copolymers, the critical $\Delta \chi$ must occur at larger values for chains with greater number of arms. In Figure S8A and B, we present the difference in the free energy between the parallel and perpendicular phases $(\Delta \mathrm{fE})$ as a function of $\Delta \chi$ for 1-, 2-, 3- and 4-arm star-block copolymers. In both cases, the transition from the perpendicular to the parallel phase occurs at larger $\Delta \chi$ values for copolymers with greater number of arms. These theoretical results clearly indicate that the entropic penalty for reorienting the n-armed starblock copolymers to parallel morphologies is larger for BCPs with larger number of arms. As a result, this theoretical conclusion provides qualitative support to our experimental observations. The effect of chain architecture on the phase behavior of n-arm star-block copolymers can be further understood by examining the distribution of the blocks in the perpendicular phase, which are revealed by the density profiles of the lamellae- and cylinder-forming systems. As shown in Figure S9A and B for the case with $\Delta \chi=0$, in both lamellae- and cylinder-forming copolymers, the microdomains extend from the substrate surface to the air interface. On the other hand, a wetting layer with parallel cylinders/lamellae at the air interface is formed at smaller $\Delta \chi$ for the 1- and 2-arm star-block copolymers, but not the 3and 4-arm copolymers. The theoretical results presented here provide complementary evidence, supporting the entropy-driven orienting effect via varying the topology of the star-copolymers. On the basis of the theoretical studies above, it is feasible to suppress the enthalpy interaction at the air/polymer interface for the formation of throughout perpendicularly oriented lamellae or cylinders in the thin-film state from the self-assembly of star-block copolymers with high surface energy core block (as shown in 
Figure 1) while there are a few layers of parallel lamellae or cylinders at the air/polymer interface due to the low surface energy of the PDMS core block.
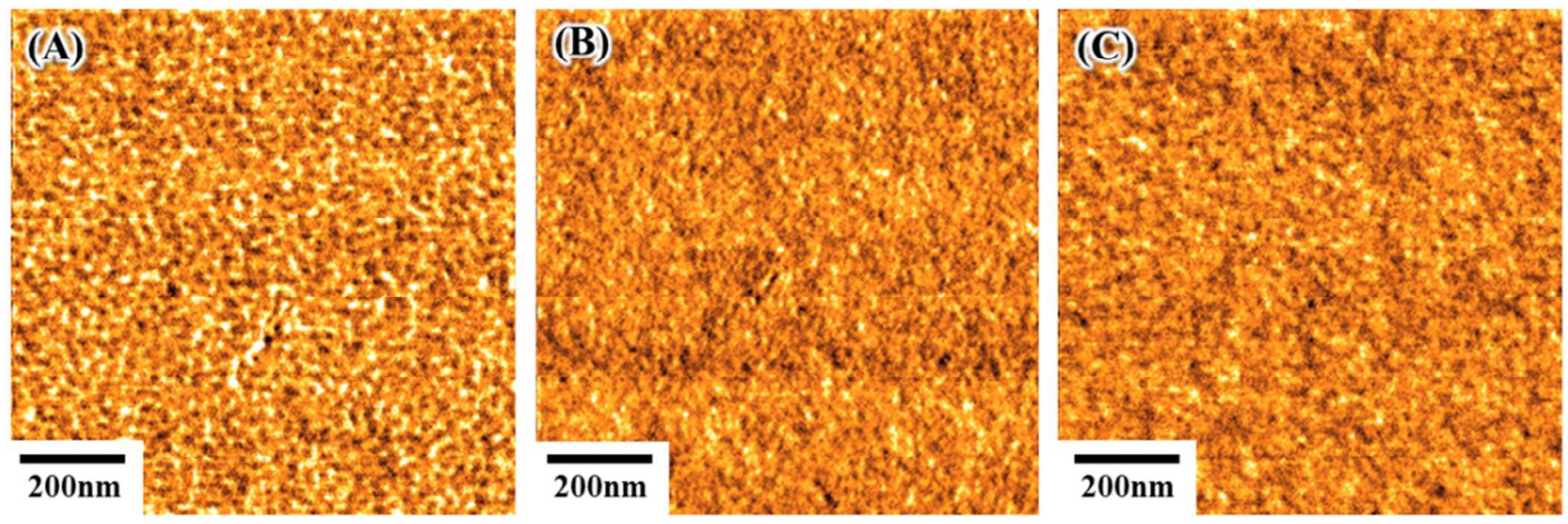

Figure S1. Tapping mode SPM top-view phase images of $\left(\mathrm{S}_{13.7}-b-\mathrm{D}_{7.11}\right)_{3}$ thin film (A) before thermal annealing; and after thermal annealing at $280^{\circ} \mathrm{C}$ for (B) $5 \mathrm{~min}$ and (C) $10 \mathrm{~min}$ with a thicknesses of $340 \mathrm{~nm}$.
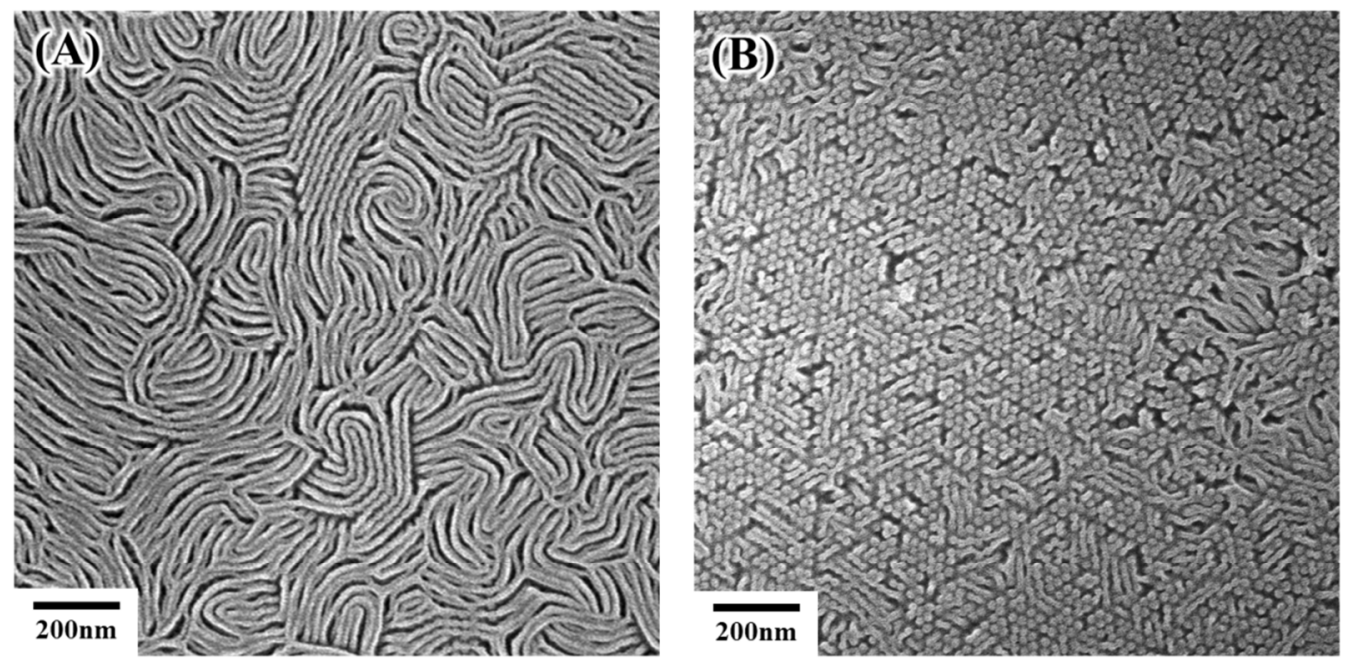

Figure S2. The $340 \mathrm{~nm}$ thick $\left(\mathrm{S}_{13.7}-b-\mathrm{D}_{7.1}\right)_{3}$ films after second round thermal annealing process by flipping the annealed film sample over, and then thermal annealing again at $280{ }^{\circ} \mathrm{C}$ for $30 \mathrm{~min}$. (A) topview (bottom side for the $1^{\text {st }}$ round) and (B) bottom-view (top side for the $1^{\text {st }}$ round) FESEM images of the thin-film sample after RIE treatment. 

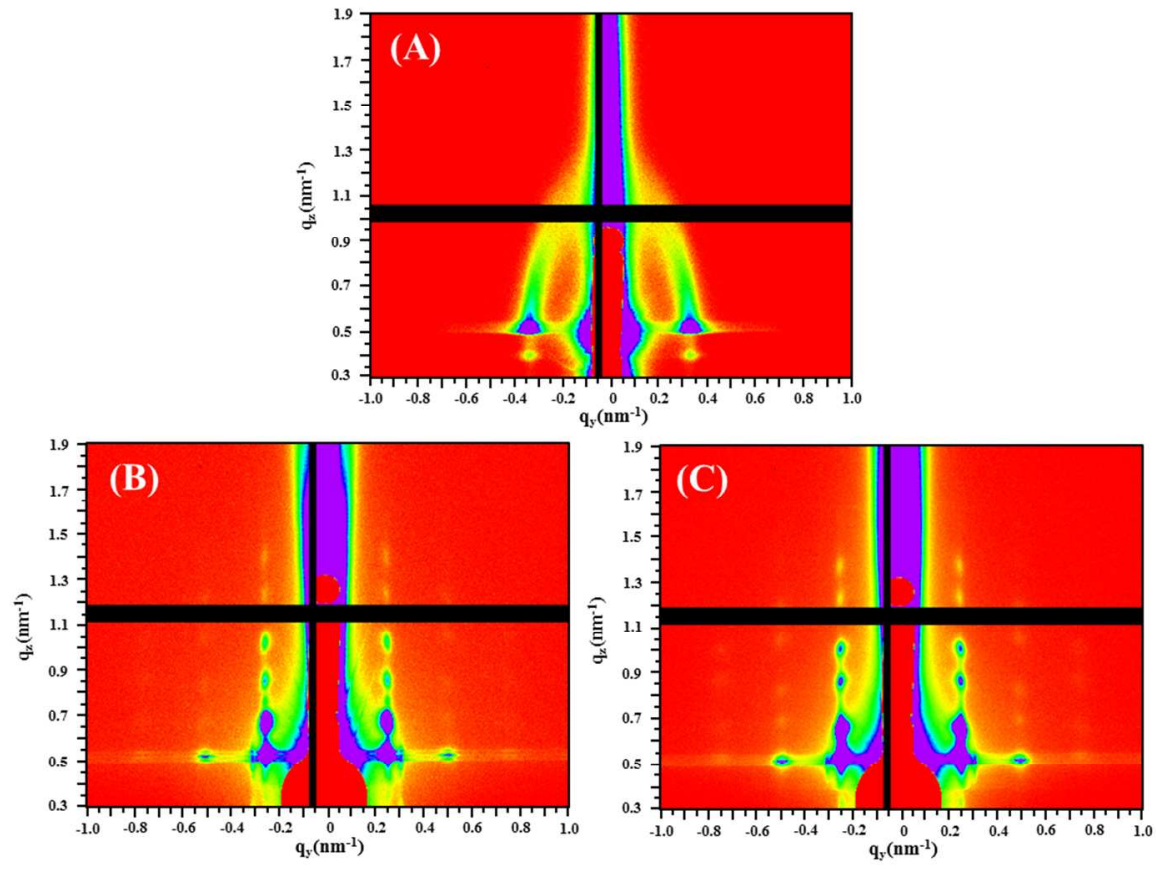

Figure S3. 2D GISAXS pattern of $\left(\mathrm{S}_{13.7}-b-\mathrm{D}_{7.11}\right)_{1}$ thin film before thermal annealing (A) and after thermal annealing at $280^{\circ} \mathrm{C}$ for 30 min with thicknesses of (B) $120 \mathrm{~nm}$, (C) $200 \mathrm{~nm}$, respectively.
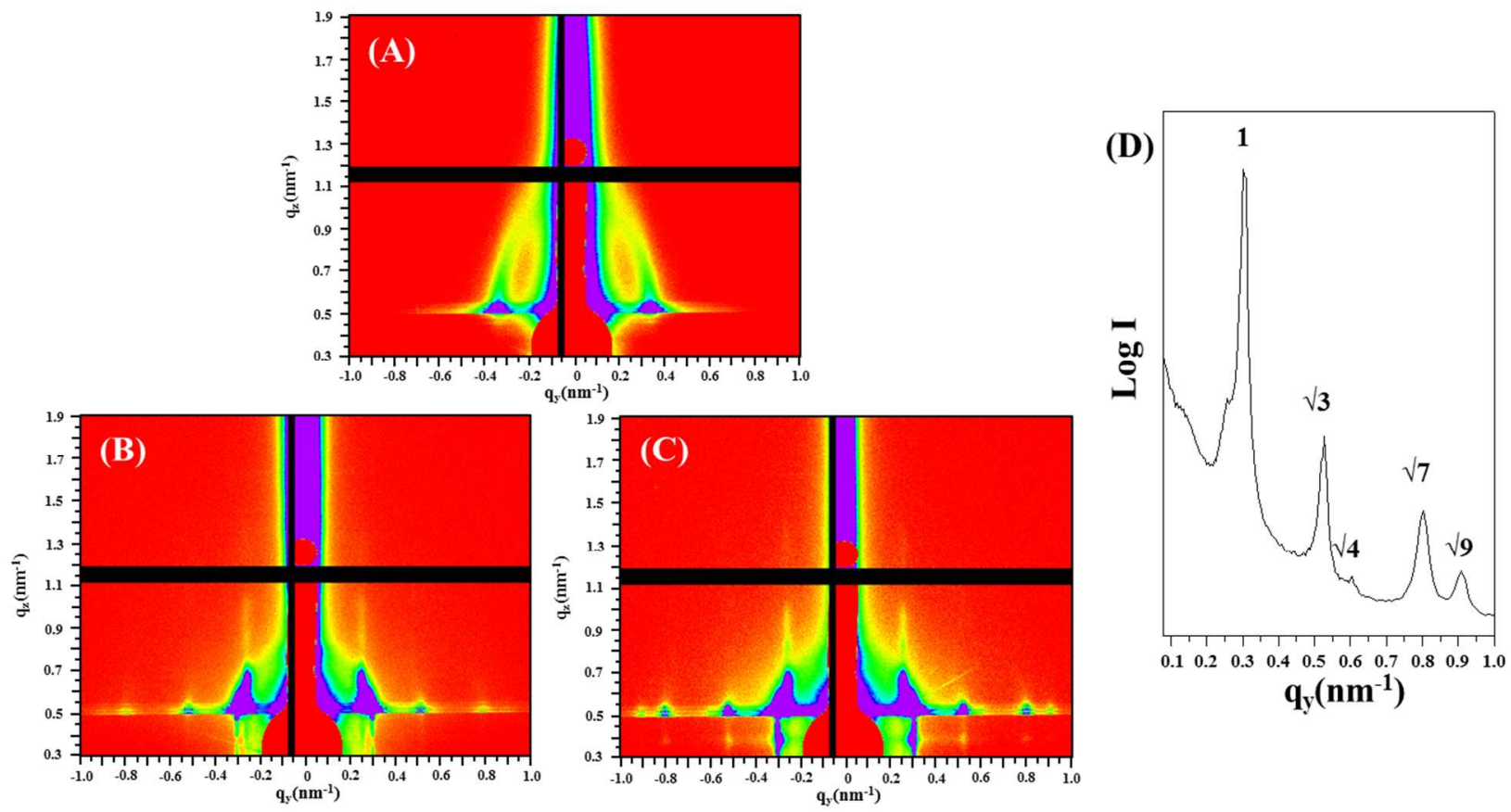

Figure S4. 2D GISAXS pattern of $\left(\mathrm{S}_{13.7}-b-\mathrm{D}_{7.11}\right)_{3}$ thin film before thermal annealing (A) and after thermal annealing at $280^{\circ} \mathrm{C}$ for 30 min with thicknesses of (B) $120 \mathrm{~nm}$, (C) 200nm, respectively. (D) $q_{y}$ scans from 2D GISAXS pattern of Fig. 3E ( $q_{y}$ scans were extracted at $\left.q_{z}=0.55 \mathrm{~nm}^{-1}\right)$. 


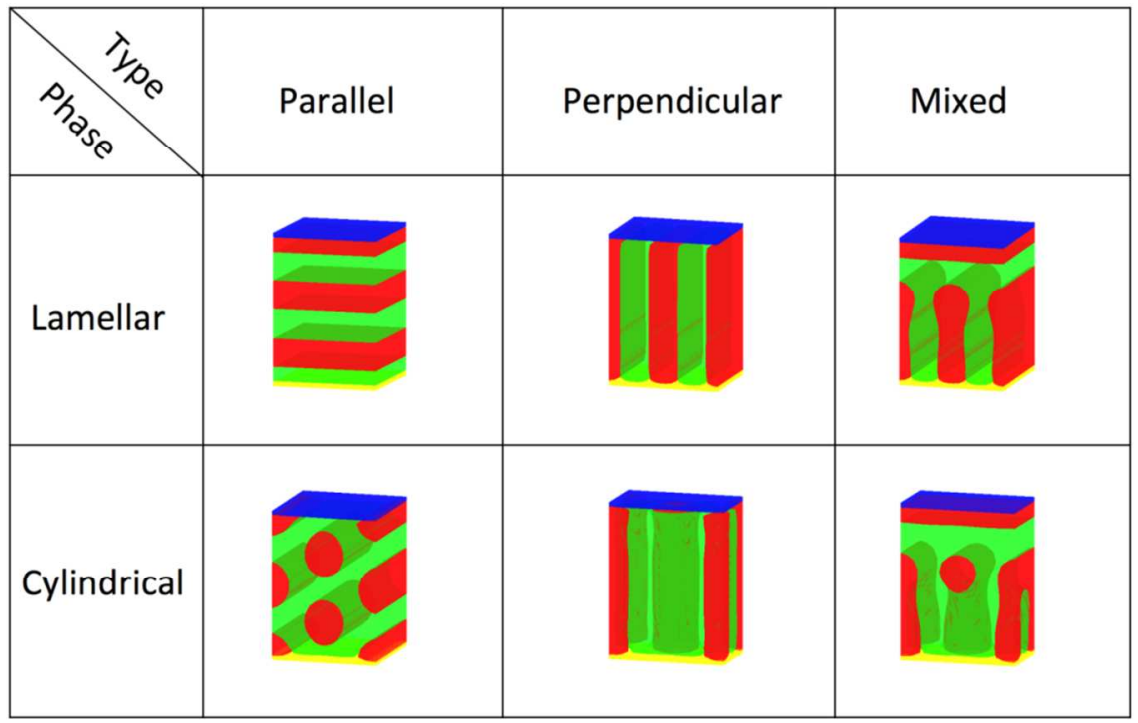

Figure S5. Density profiles demonstrating the parallel, perpendicular and mixed, lamellae- and cylinder-forming morphologies. In the above density profiles, the A- and B-species are represented using the red and green colors, respectively. Similarly, the polymer-air and polymer-substrate interfaces are shown using blue and yellow colors.
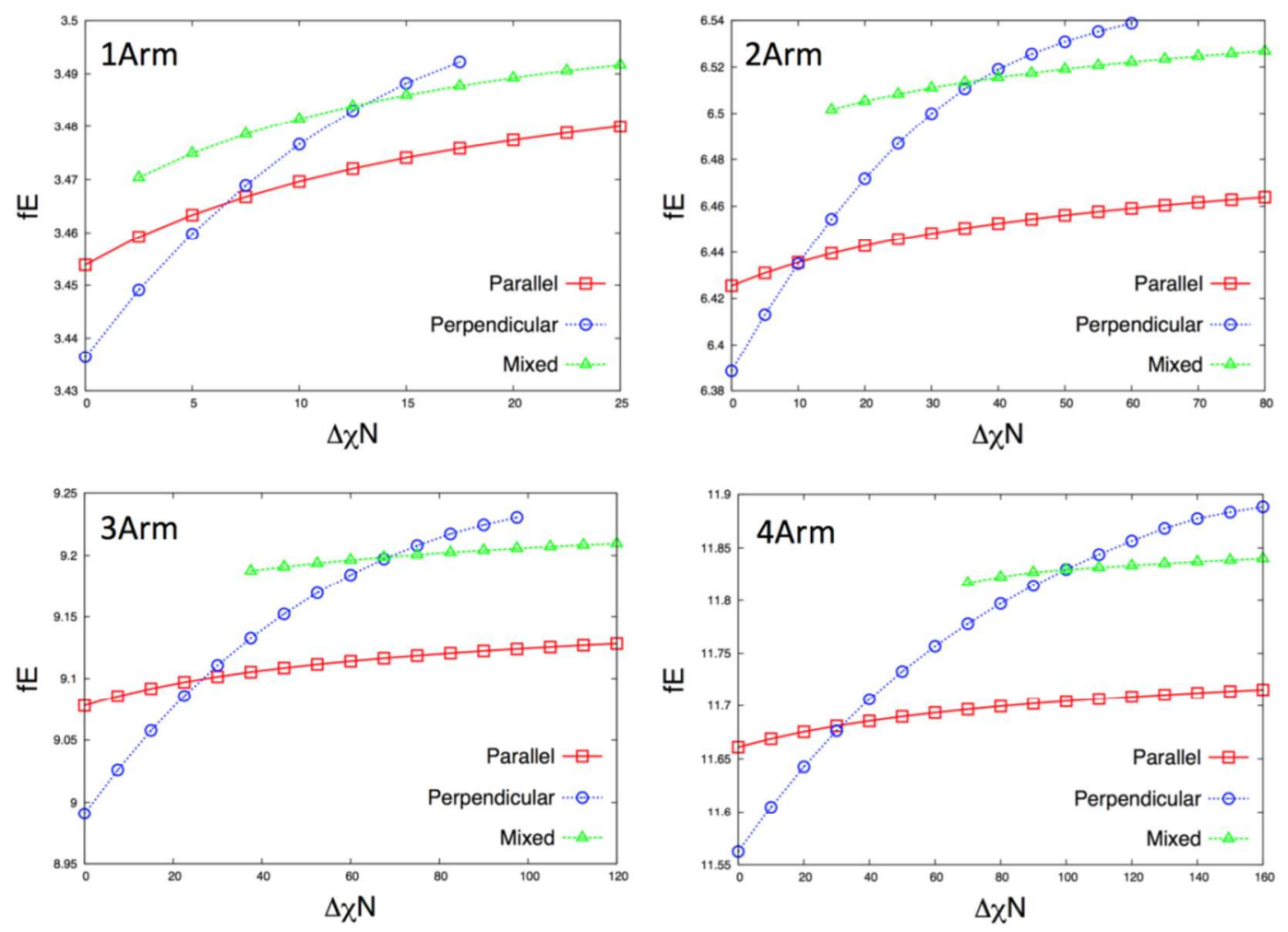

Figure S6. Free energy calculated using SCFT as a function of $\Delta \chi=\chi_{\mathrm{BAir}} \chi_{\mathrm{AAir}}$ for the lamellae-forming 1-, 2-, 3- and 4-arm star-block copolymers. We plot the free energy for the parallel, perpendicular and mixed phases for a system with $f_{A}=0.5, \chi_{A B}=0.14, \chi_{A S u b}=0.08, \chi_{B S u b}=0.06$ and $h=2 d_{o}$. 

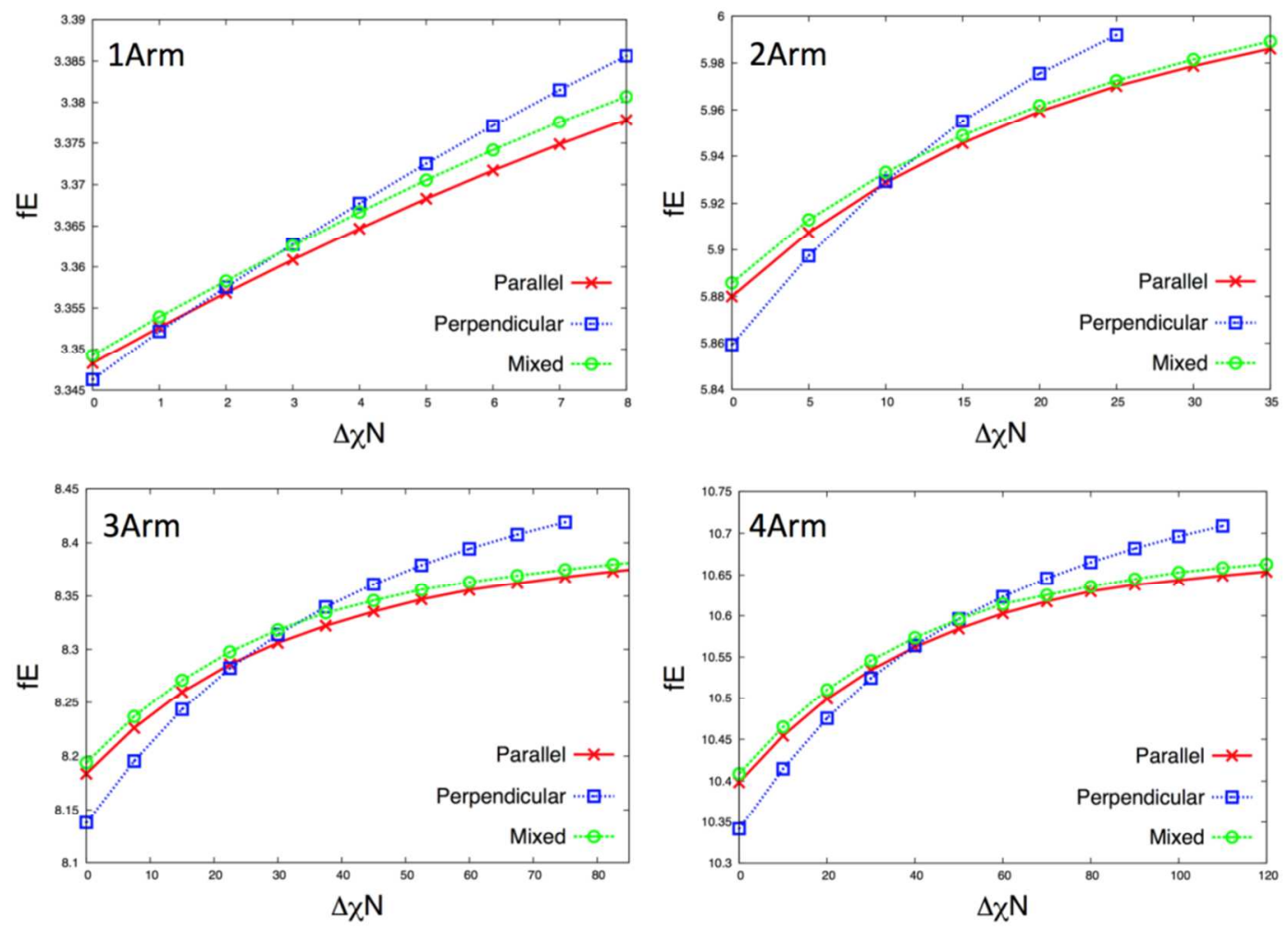

Figure S7. Free energy calculated using SCFT as a function of $\Delta \chi=\chi_{\text {BAir- }} \chi_{\text {AAir }}$ for the cylinder-forming 1, 2-, 3- and 4-arm star-block copolymers. We plot the free energy for the parallel, perpendicular and mixed phases for a system with $f_{A}=0.35, \chi_{A B}=0.14, \chi_{A S u b}=0.08, \chi_{B S u b}=0.06$ and $h=2 d_{o}$.
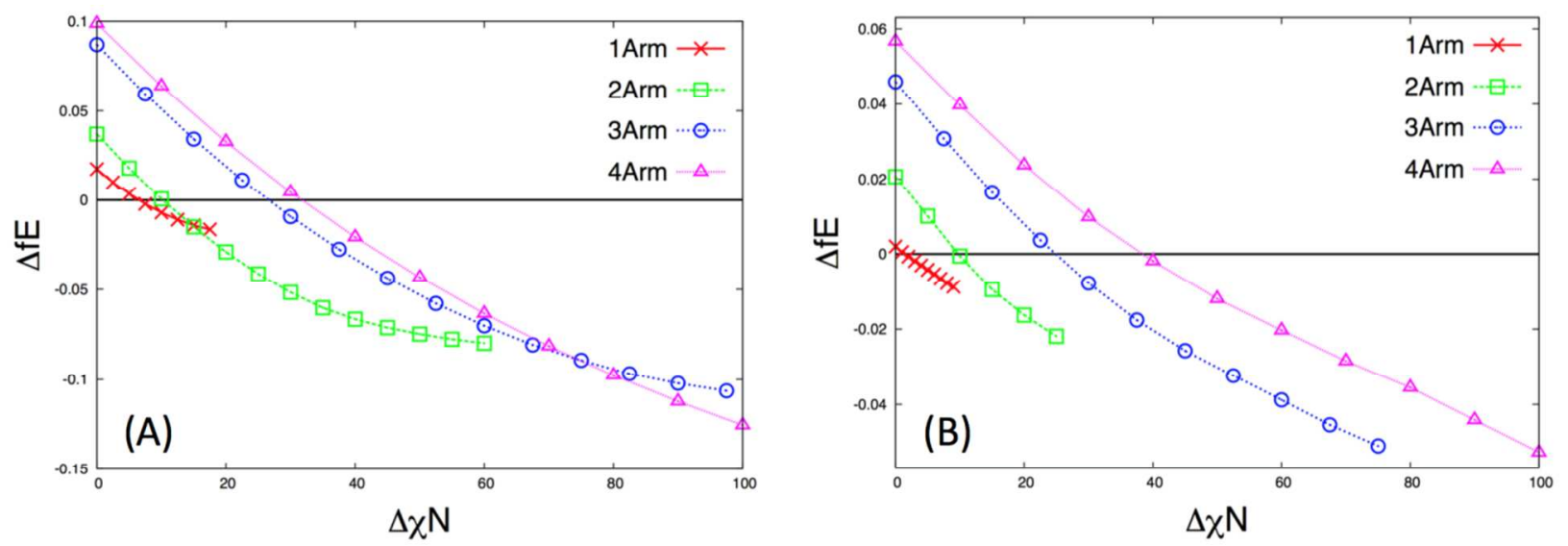

Figure S8. The free energy difference between the parallel and perpendicular phase for (A) the lamellaeand (B) cylinder-forming $\mathrm{n}$-arm star-block copolymer thin films with $\mathrm{h}=2 \mathrm{~d}_{0}$. Here, $\Delta \mathrm{f} \equiv \mathrm{f}_{\text {parallel }}-$ $\mathrm{f}_{\text {perpendicular }}$ plotted as a function of $\Delta \chi \equiv \chi_{B A i r}-\chi_{A A i r}$. 

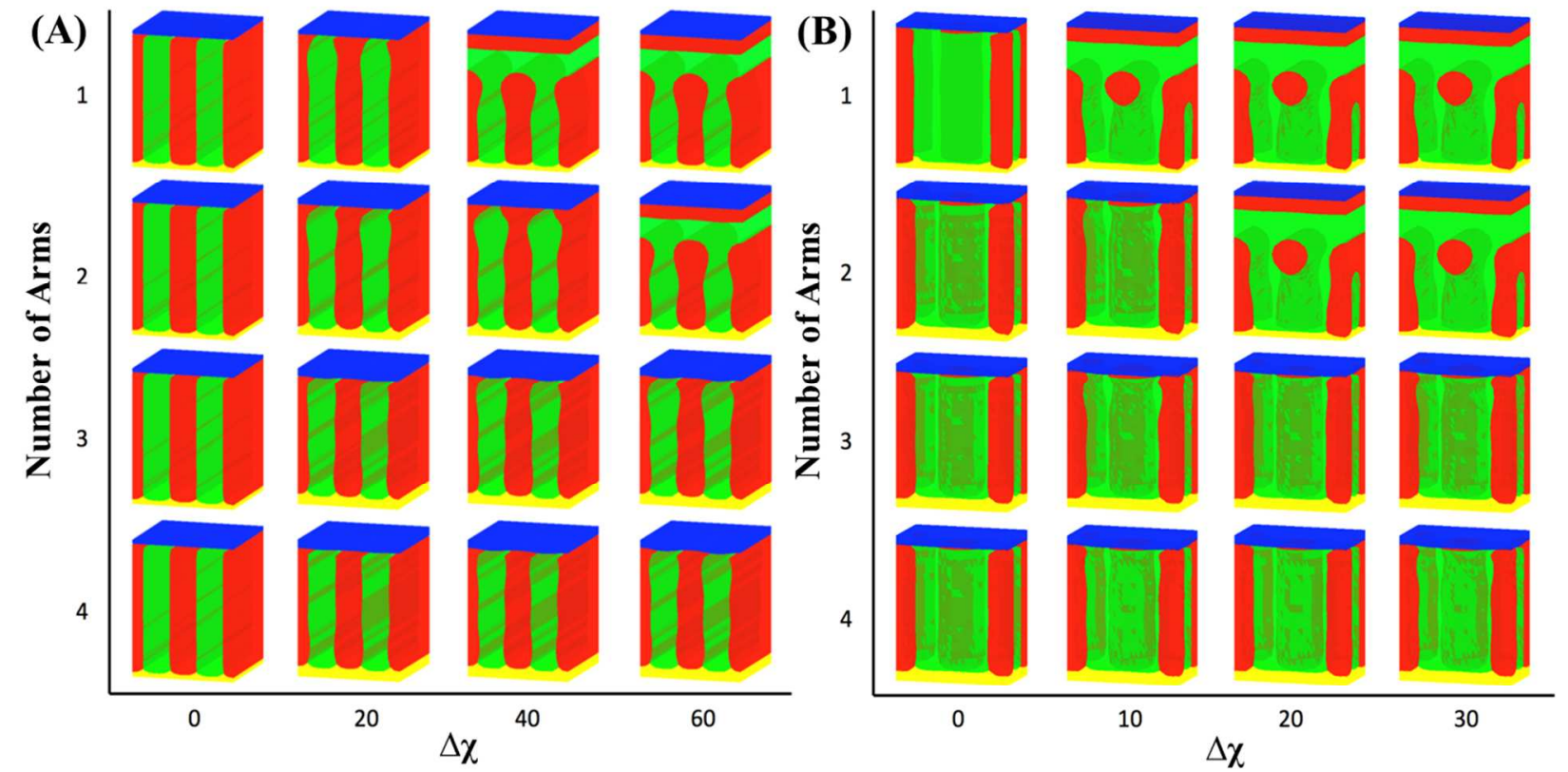

Figure S9. 3-D density profiles for the (A) lamellae-forming and (B) cylinder-forming 1, 2, 3 and 4 arm star-block copolymers as a function of $\Delta \chi$.

\section{References}

1. Wu, S. Polymer Interface and Adhesion; Marcel Dekker: New York, 1982; Chapter 5

2. Mark, J. E. Physical Properties of Polymers Handbook; Woodbury, New York, 1996; 669-677

3. Moreira, J. C. Influence of temperature, molecular weight, and molecular weight dispersity on the surface tension of PS, PP, and PE. I. Experimental Journal of Applied Polymer Science 2001, 82, 1907-1920.

4. Roe, R. J. Surface tension of polymer liquids J. Phys. Chem 1968, 72, 2013-2017.

5. Shi, A.-C., Self-consistent Field Theory of Block Copolymers, in Developments in Block Copolymer Science and Technology, Edited by I. W. Hamley (Wiley, New York, 2004), Chap. 8.

6. Fredrickson, G. H. The Equilibrium Theory of Inhomogeneous Polymers (Clarendon, Oxford, 2006).

7. Shi, A. C., Li, B. Self-assembly of diblock copolymers under confinement Soft Matter 2013, 9, 1398-1413. 